\title{
VOCÊ DIZ QUE QUER UMA REVOLUÇÃO: TODOS QUEREMOS MUDAR O MUNDO
}

\section{RESUMO}

O livro "Leading the Revolution" ou "Liderando a Revolução", de Gary Hamel, traz importantes contribuições para os estudos de inovação quando associados à estratégia. $\mathrm{O}$ autor afirma que estamos no limiar da era da revolução em que as formas tradicionais de buscar desempenho para as organizações não funcionam mais. A marca desta nova era, segundo Gary Hamel, é a inovação radical, cuja característica mais importante é fazer diferença para o cliente, gerando valor para a organização e prepará-la para o futuro, se necessário, mudando seu modelo de negócio. Este trabalho, além de discutir algumas obras de Gary Hamel, "Liderando a Revolução", em especial, investiga a influência do livro em obras de outros autores por meio de técnicas bibliométricas. Identificamos quatro abordagens adotadas pelos autores que usam "Liderando a Revolução" como referência: gestão de mudanças; empreendedorismo; estratégia e competitividade e inovação. Este trabalho oferece uma análise do impacto de Liderando a Revolução e traz caminhos para o uso da teoria de Hamel em estudos futuros de inovação e estratégia.

Palavras-chave: Liderando a Revolução; Gary Hamel; Inovação.

\section{YOU SAY YOU WANT A REVOLUTION: WE ALL WANT TO CHANGE THE WORLD}

\begin{abstract}
The book "Leading the Revolution", by Gary Hamel, brings important contributions to innovation studies when associated with the strategy. The author states that we are on the threshold of revolution era in which traditional forms of performance' search for organizations no longer work. The milestone of this new era, according to Gary Hamel, is the radical innovation, whose most important feature is making difference for the customer, creating value for the organization and preparing it for the future, through changing its business model, if necessary. This paperwork, besides discussing some of Gary Hamel's works, "Leading the Revolution", in particular, investigates the influence of this book in other authors' work, through bibliometric techniques. We have identified four approaches adopted by authors that use "Leading the Revolution" as a reference: change management; entrepreneurship; strategy and competitiveness and innovation. This work provides an analysis of the impact of "Leading the Revolution" and brings ways for the use of Hamel's theory in future studies of innovation and strategy.
\end{abstract}

Keywords: Leading the Revolution; Gary Hamel; Innovation. 


\section{USTED DICE QUE QUIERE UNA REVOLUCIÓN: TODOS QUEREMOS CAMBIAR EL MUNDO}

\section{RESUMEN}

El libro "Liderando la Revolución" o "líder de la Revolución" por Gary Hamel, aporta importantes contribuciones a los estudios de innovación cuando se asocia con la estrategia. El autor afirma que estamos en el umbral de la era de la revolución en la que las formas tradicionales de rendimiento de la búsqueda para las organizaciones ya no funcionan. El sello distintivo de esta nueva era, según Gary Hamel es la innovación radical, la característica más importante es hacer una diferencia para el cliente, la creación de valor para la organización y prepararlo para el futuro, si es necesario, cambiar su modelo de negocio. Este trabajo, y discutir algunas obras de Gary Hamel, "dirigir la revolución", en particular, investigar la influencia del libro en las obras de otros autores a través de técnicas bibliométricas. Identificado cuatro enfoques adoptados por los autores que utilizan "dirigir la revolución" como referencia: la gestión del cambio; la iniciativa empresarial; estrategia y la competitividad y la innovación. Este trabajo ofrece un análisis del impacto de la dirección de la revolución y trae maneras para que el uso de la teoría de Hamel en futuros estudios de la innovación y la estrategia.

Palabras clave: Líder de la Revolución; Gary Hamel; La Onnovación.

\footnotetext{
${ }^{1}$ Doutorando em Administração pelo Programa de Pós Graduação em Administração da Universidade Nove de Julho PPGA/UNINOVE. Brasil. E-mail: luizguerrazzi@hotmail.com

${ }^{2}$ Doutorando em Administração pelo Programa de Pós Graduação em Administração da Universidade Nove de Julho PPGA/UNINOVE. Brasil. E-mail: ooluis@gmail.com

${ }^{3}$ Doutorando em Administração pelo Programa de Pós Graduação em Administração da Universidade Nove de Julho PPGA/UNINOVE. Brasil. E-mail: christianfalaster@gmail.com
} 


\section{INTRODUÇÃO}

As contribuições do livro "Leading the Revolution", ou "Liderando a Revolução", são importantes para os estudos de inovação quando tangem a estratégia. Particularmente, Gary Hamel traz importantes contribuições para a dinâmica de competitividade das empresas quando a inovação estudada é do tipo radical. Neste estudo, construímos uma resenha crítica com base bibliométrica para analisar o impacto do livro Liderando a Revolução na literatura de inovação.

A importância de "Liderando a Revolução" está alicerçada em sua lógica acerca das inovações radicais. Segundo Gary Hamel (2000), só chegarão ao futuro as empresas que adotarem uma estratégia baseada em inovações radicais e que tenham dinamismo e flexibilidade para alterar seus modelos de negócio com a velocidade requerida pelo ambiente. O futuro será de quem se beneficiar das inovações tecnológicas para redirecionar a estratégia. Empresas tradicionais que não estimulam a inovação conseguirão desempenho satisfatório, mas sem sustentabilidade, logo serão ultrapassadas.

O foco deste trabalho está no livro de Gary Hamel, "Liderando a Revolução" (Leading the Revolution) publicado em 2000 e em sua obra, de modo mais geral. Neste artigo foram empregadas técnicas bibliométricas em trabalhos coletados no portal Web of Science da Thomson-Reuters. Os artigos da amostra foram submetidos às análises de citação e de pareamento bibliográfico. Uma análise fatorial exploratória também foi executada nos resultados do pareamento bibliográfico com o objetivo de agrupar trabalhos com aspectos conceituais comuns (Lin, \& Cheng, 2010).

Contribuímos neste estudo para duas vertentes. Em primeiro lugar, uma análise aprofundada do impacto de "Liderando a Revolução" pode trazer evidências para o uso da teoria de Hamel em estudos futuros de inovação e estratégia (quando fortemente ligada à inovação). Em segundo lugar, este estudo contribui por trazer a obra de Hamel para estudantes, principalmente de mestrado e doutorado, como material de consulta para conhecerem um pouco sobre o papel da inovação radical na competitividade das empresas.

Esta resenha está estruturada em mais três partes além desta introdução. Na revisão de literatura, apresentamos o pensamento de Gary Hamel abordando algumas de suas obras mais emblemáticas em sua carreira e abordamos com mais profundidade seu livro "Liderando a Revolução". Descrevemos a metodologia utilizada na seção de método e a seguir mostramos a influência de "Liderando a Revolução" na produção acadêmica sobre inovação e estratégia, seguido de discussão e conclusões.

\section{REVISÃO DE LITERATURA}

Nesta seção, apresentamos um pouco do histórico de publicações de Gary Hamel para contextualizar o leitor. Trazemos também uma síntese das principais ideias da obra Liderando a Revolução. Finalizamos a seção explicando um pouco do pensamento de Hamel (2000) acerca da inovação radical.

\subsection{O pensamento de Gary Hamel}

A trajetória que Gary Hamel traçou durante sua carreira o levou a ser um dos principais pensadores em gestão empresarial do mundo. Juntamente com C. K. Prahalad publicou, em 1990, um estudo em que mergulhava no âmago da empresa para mostrar que, mais do que de produtos e de serviços, o que dá identidade à empresa são suas competências essenciais (Prahalad, \& Hamel, 1990), este trabalho influenciou diretamente sua obra futura. É por meio de suas competências essenciais que a empresa desenvolve capacidades para explorar recursos e adquirir vantagens estratégicas que a farão ter desempenho superior. Segundo seu trabalho posterior, as empresas são consideradas como conjuntos de competências e capacidades (Hamel, \& Prahalad, 1995), indo ao encontro da visão de que a empresa é um conjunto de recursos (Penrose, 1959) e da teoria baseada em recursos (RBV). Inclusive, seu trabalho com Prahalad de 1990 é tido como um dos principais dentro do campo da RBV (Ramos-Rodríguez \& Ruíz-Navarro, 2004; Serra, Ferreira, Almeida, \& Vanz, 2012)

A RBV sustenta que a heterogeneidade das empresas determina vantagens competitivas em função do uso dos recursos de que dispõe (Barney, 1991). Esses recursos são explorados ou buscados pelas capacidades da empresa, que asseguram a assimetria de desempenho devido às capacidades essenciais de cada uma, entendidos como a condição que possuem de selecionar, combinar, buscar, desenvolver e integrar recursos (Prahalad, \& Hamel, 1990; Teece, Pisano, \& Shuen, 1997). Essas competências contribuem para adicionar valor aos clientes, gerar vantagens competitivas e sustentar o modelo de negócios (Hamel, \& Prahalad, 1995; Teece, 2010)). Estas competências são desenvolvidas ao longo do tempo devido a um processo de acúmulo e aprendizagem (Hoskinsson, Hitt, Ireland, \& Harrison, 2009), sendo que este histórico será determinante para a criação de novas competências e capacidades (Teece, Pisano, \& Shuen, 1997).

As competências da empresa podem levá-la a criar formas inteiramente novas de vantagens competitivas de modo a redefinir seu modelo de negócios, preparando-a para o futuro. Uma preocupação recorrente dos gestores é fazer com que sua empresa chegue ao futuro primeiro; o desenvolvimento da estratégia deve expressar a 
transição da empresa de sua posição atual para um estado futuro desejável (Mintzberg, 1994). Gary Hamel e C. K. Prahalad usam sua visão das competências essenciais da empresa para indicar como prepará-la para o futuro, mantendo seu desempenho e prolongando sua vida (Hamel, \& Prahalad, 1995).

Em "Competindo pelo Futuro" (Hamel \& Prahalad, 1995), os autores afirmam que a corrida para o futuro ocorrerá em três estágios. Em primeiro lugar, haverá a competição pela liderança intelectual, relacionada às inovações tecnológicas. Em segundo lugar, uma competição pelo caminho a seguir, relacionado à formulação do modelo de negócio e em terceiro lugar, a competição pelo posicionamento na cadeia de valor, considerando uma rede estratégica formada por fornecedores e clientes.

\subsection{Liderando a Revolução}

Gary Hamel (2000) propõe um plano de ação para transformar indivíduos e empresas em revolucionários. Empresas revolucionárias estão pulverizando os velhos modelos de negócio e propondo novos modelos totalmente inovadores. Para ter desempenho superior na era da revolução, as inovações radicais precisam estar continuamente no foco das empresas. Baseado nisso, o livro propõe um caminho para o futuro abordando quatro aspectos. Primeiro, define a inovação radical e explica os princípios subjacentes a elas. Segundo, analisa a criação e as características dos novos e revolucionários modelos de negócio. Terceiro, identifica os principais fatores organizacionais que tornam a empresa preparada para a revolução. Quarto, discorre sobre as condições que transformam a inovação em vantagem sustentável das empresas.

Em "Liderando a Revolução", Hamel (2000) afirma que empresas têm tentado várias ações para tornarem-se mais eficientes. Promoveram cortes de pessoal e de despesas, reengenharias e integração de processos e de unidades. Porém, este arsenal de ações utilizado teve sua eficácia exaurida. Se quiserem gerar mais riqueza, ou até mesmo sobreviver, a única saída para as empresas está nas inovações radicais. Com o receituário tradicional, as grandes corporações tornaram-se muito parecidas e só as empresas que criarem produtos e modelos de negócio revolucionários, serão capazes de prosperar no futuro. Hamel (2000) sustenta que a era do progresso acabou; estamos no limiar de uma nova era, a era da revolução.

A revolução, segundo Hamel (2000), passa por uma transformação no interior das empresas, em sua organização e na participação de seus funcionários. Afirma ainda que impor regras de cima para baixo é um sistema lento demais para reagir ao ritmo das mudanças e que o envolvimento de todos num processo sistemático de criação é o caminho. Hamel (2000) pondera que o papel do líder é cada vez menos tomar grandes decisões e cada vez mais ser um arquiteto serial, criando um ambiente que motive a contribuição e a colaboração, com menos hierarquia. O papel do novo líder também será o de criar condições para que a empresa adicione uma nova competência essencial, que seja capaz de motivar todos a gerarem novas ideias e, por meio delas, se recriar continuamente.

\section{A inovação radical}

Em "Liderando a revolução", o foco não é a inovação radical em si, mas reforça o papel da adoção de um modelo de negócio e de uma estrutura organizacional que gerem continuamente inovações radicais. A inovação radical precisa simultaneamente gerar mais riqueza para a empresa e representar uma diferença para o cliente, Segundo Hamel (2000), sua principal característica é a geração de valor. A inovação radical pode ocorrer de dois modos: ela pode apenas alterar um componente do negócio ou do produto ou pode transformar todo o modelo de negócio. Nem todas as inovações radicais vão gerar uma revolução, mas em toda empresa é desejável haver um esforço em gerar inovações radicais em todos os níveis hierárquicos, numa quantidade suficiente para aumentar suas chances de criar aquilo que vai transformar radicalmente seu perfil.

Empresas muito tradicionais, com estrutura muito hierarquizada são empecilhos para a inovação. Necessitam passar por uma transformação, levando-as a criar uma estrutura mais igualitária para a estratégia e inovação. As inovações devem surgir de qualquer nível da hierarquia. Em seu modelo para a nova empresa, disciplina e liberdade não são mutuamente excludentes, afirma Hamel (2000), e transparência, meritocracia e colaboração devem ser buscados.

\section{Modelos de negócio revolucionários}

Para Hamel, a inovação no conceito de negócio consiste em introduzir a visão estratégica, indo além da inovação incremental, mas envolvendo o conceito de um negócio como um todo com o foco em idéias e em práticas inovadoras. Isto faz com que a inovação no conceito de negócio seja mais abrangente do que a inovação focada somente em produtos ou tecnologia. Modelos de negócio revolucionários baseiam-se em inovações nos conceitos do negócio: revisões radicais nos modelos de negócios existentes, de forma a criar novo valor para os clientes, para os competidores e mais riqueza para os investidores.

A necessidade para a inovação no conceito de negócio origina-se em parte pela diminuição dos retornos das várias formas tradicionais de revisão do modelo - reengenharia, corte de custos, racionalizações e etc - que focam na manutenção ao invés da mudança na forma de fazer negócios. As empresas podem remodelar-se em organizações inovadoras, pelo engajamento contínuo de sua estrutura num ciclo de geração de idéias, experimentos, acessos e implementações. 


\section{Empresa preparada para a revolução}

Organizações que atuam na defensiva, lutando por adaptar seu modelo de negócio à inovação do conceito de negócio de outrem não estão preparadas para o futuro e não terão um desempenho acima da média. Como então preparar a organização para a revolução? Hamel propõe um roteiro em oito passos:

1 - Construa um ponto de vista;

2 - Escreva um manifesto;

3 - Crie uma coalizão;

4 - Escolha seus alvos e seus momentos;

5 - Coopte e neutralize;

6 - Ache um tradutor;

7 - Vença pequeno, vença antes e vença muitas vezes;

8 - Isole, infiltre e integre.

\section{A inovação radical como forma de vantagem competitiva sustentável}

O conceito de inovação em negócio por meio da capacidade de imaginar conceitos de negócios radicalmente diferentes ou novas formas radicais de diferenciar os conceitos existentes de negócios, tornarse-á a vantagem competitiva definidora na era da revolução. Empresas autoritárias e orientadas para o controle continuarão a cair, enquanto as "democratas" da informação, criativas e avessas à autoridade, serão as empresas do futuro.

Segundo Hamel, a singularidade na forma de pensar o negócio é um componente que leva a empresa a sobressair-se. Quanto maior for a convergência entre os modelos de negócio, menor a chance de lucro. Deve-se, portanto, criar um modelo de negócio que seja único em sua concepção e execução e para fazê-lo, é preciso determinar os pontos de diferença ao longo dos componentes principais do conceito e dos benefícios que tais pontos vão oferecer aos stakeholders.

\section{MÉTODO}

A bibliometria é uma análise estatística da produção científica que auxilia na compreensão de como campos de pesquisa, disciplinas e publicações se relacionam, sendo indicada para identificar o modo como a área está estruturada, as principais abordagens utilizadas e as principais obras (Pritchard 1969; Vogel, \& Güttel, 2013; Zupic, \& Čater, 2015). Neste trabalho empregamos técnicas bibliométricas para investigar a influência de Liderando a Revolução por meio da identificação das obras que adotaram o livro como referência. Usamos a análise de citação e o pareamento bibliográfico combinado com uma análise fatorial exploratória.

A análise de citação baseia-se na contagem de vezes em que uma determinada obra é citada por outros acadêmicos. $\mathrm{O}$ conceito subjacente é que são citados apenas artigos que têm relação com determinado trabalho e, portanto, quanto mais citados, mais influenciam a pesquisa sobre o assunto (RamosRodrigues, \& Ruiz-Navarro, 2004; Tahai, \& Meyer, 1999). O pareamento bibliográfico mede a utilização de uma mesma referência por duas ou mais obras. Parte do pressuposto de que quanto maior o número de citações compartilhadas, maior a similaridade entre elas (Vogel, \& Güttel, 2013). Empregamos o pareamento neste trabalho, pois ele permite um exame do que tem sido produzido sobre o tema, portanto a linha de frente da pesquisa (Zupic, \& Cater, 2015). Este método difere de técnicas mais tradicionais que analisam as referências empregadas, fixando o foco nas bases com que o tema é pesquisado (Vogel, \& Güttel, 2013).

Finalmente, conduzimos uma análise fatorial exploratória com o objetivo de identificar fatores que representem aspectos conceituais comuns relativos às abordagens das obras com que Liderando a Revolução é usado como referência. Os artigos são agrupados de acordo com sua proximidade conceitual usando como medida de similaridade a quantidade de vezes em que são pareados, isto é, usam a mesma referência. Os fatores mais representativos são os que possuem a maior variância explicada (Shafique, 2013). A carga fatorial de um documento em um dado fator representa o quanto ele se adequa ao mesmo.

\subsection{Coleta de dados e amostra}

Os dados foram obtidos na base de dados Web of Science, da Thomson Reuters (www.webofknowledge.com). Essa base foi escolhida por sua abrangência e por disponibilizar os dados em um formato que otimiza o esforço de coleta e operacionalização. Por meio de sua ferramenta de busca, foram identificados trabalhos que usaram "Leading the Revolution" como referência. Não foi estipulado limite temporal, assim, participaram da coleta todos os artigos listados no portal.

Foram identificados 347 trabalhos publicados entre 2001 e 2016. Estes trabalhos foram validados por meio da leitura de seu título, resumo e introdução. Descartamos trabalhos que não tratavam de temas relacionados ao livro. A Figura 1 traz a quantidade de artigos publicados por ano de nossa amostra. Embora a amostra contenha trabalhos publicados até junho de 2016, este mesmo ano não foi contemplado no gráfico por não ser um período completo, porém os documentos foram considerados na análise de citação e de pareamento. A amostra contém 5 trabalhos para o ano de 2016 até a data da coleta. Pode-se notar a constante influência de Liderando a Revolução ao longo dos anos; apesar do livro estar com mais de 15 anos de sua publicação, continua sendo adotado em novas obras (Figura 1). 
Figura 1 - Evolução das publicações

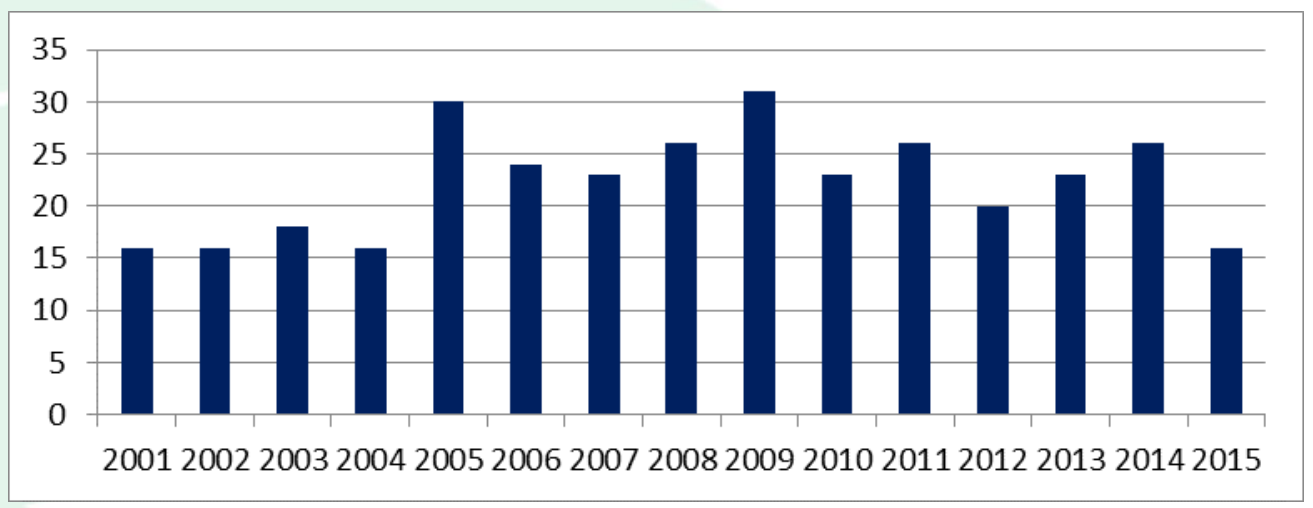

\subsection{Procedimentos de análise}

Neste estudo, foram executadas as análises de citação e de pareamento bibliográfico combinada com uma análise fatorial exploratória para classificação e ordenação dos dados. Os procedimentos bibliométricos foram conduzidos para os 66 artigos mais citados da coleção inicial de 342 trabalhos. As obras escolhidas por ordem decrescente de citação, representam juntas $80 \%$ do total de citações das 342 obras. Primeiro, foi executada a análise de citação, baseada na extração da referência e contagem de vezes que a mesma é citada por outros trabalhos constantes da amostra. A segunda análise foi a de pareamento bibliográfico, cuja frequência de co-ocorrência, após tratamento com o Microsoft Excel é o input para a análise fatorial exploratória. As análises de citação e de pareamento foram conduzidas com o software Bibexcel (Pilkington, 2006).

O terceiro procedimento foi a execução da análise fatorial exploratória, realizada com o software SPSS (versão 20), baseada nos pareamentos obtidos. Utilizou-se a rotação varimax por ser a que oferece um resultado mais facilmente interpretável (Fabrigar, Wegener, MacCallun, \& Strahan, 1999). Considerou-se apenas as cargas fatoriais maiores que 0,40 , como prérequisito para a variável pertencer a um fator (Shafique, 2013). Esta condição resultou na classificação de 50 trabalhos em quatro grupos e, por meio da análise de cada componente, é validada a formação do fator e este é nomeado (Lin, \& Cheng, 2010).

\section{RESULTADOS E DISCUSSÃO}

\subsection{Análise de citações}

Os 66 artigos da amostra utilizaram-se de cerca de 4000 referências. A Tabela 1 contém as obras mais citadas por período. As colunas trazem a quantidade de citações em valor absoluto e relativo, considerando a quantidade de trabalhos publicados em todo o período considerado. Por exemplo, Hamel (2000) foi citado 66 vezes no período total, ou em $100 \%$ dos trabalhos, já que, por constituição, toda a amostra é composta de artigos que o citam.

Vale notar que as obras mais citadas dizem respeito ao desenvolvimento de capacidades pela empresa. Desenvolver capacidades para alargar as fronteiras da empresa com o intuito de aumentar desempenho e sobreviver. Esse fato está expresso em Teece, Pisano e Shuen (1997) sobre a competência essencial da empresa de forjar novas capacidades como reação às mudanças contextuais. A adequação da estratégia para visar a prospecção e captação de novas oportunidades está em Zahra e Covin (1995) e Covin e Slevin (1990). Referências da teoria de recursos também são usados (Barney, 1991; March, 1990; Wernerfelt, 1984 e Penrose, 1959; Cohen e Levinthal, 1990). Esses trabalhos, de modo geral, preconizam que a empresa precisa usar eficientemente seus recursos para obter vantagens competitivas. 
Você Diz Que Quer Uma Revolução: Todos Queremos Mudar o Mundo

Tabela 1 - Frequência de citações

\begin{tabular}{|l|c|c|}
\hline & $\mathbf{2 0 0 1}$ a 2015 & \% \\
\cline { 2 - 3 } & $\mathbf{N}$ & $100 \%$ \\
\hline Hamel (2000) & 66 & $30 \%$ \\
\hline Teece, Pisano e Shuen (1997) & 14 & $21 \%$ \\
\hline Barney (1991) & 10 & $15 \%$ \\
\hline Hamel e Prahalad (1994) & 10 & $15 \%$ \\
\hline Nelson e Winter (1982) & 10 & $15 \%$ \\
\hline March (1991) & 9 & $14 \%$ \\
\hline Zahra e Covin (1995) & 9 & $14 \%$ \\
\hline Cohen e Levinthal (1990) & 9 & $14 \%$ \\
\hline Eisenhardt e Martin (2000) & 8 & $12 \%$ \\
\hline Covin e Slevin (1989) & 8 & $12 \%$ \\
\hline Porter (1980) & 7 & $11 \%$ \\
\hline Utterback (1994) & 7 & $11 \%$ \\
\hline Zahra (1991) & 7 & $11 \%$ \\
\hline Wernerfelt (1984) & 7 & $11 \%$ \\
\hline Penrose (1959) & 7 & $11 \%$ \\
\hline Shane e Venkataraman (2000) & 7 & $11 \%$ \\
\hline Miller e Friesen (1982) & 7 & $11 \%$ \\
\hline Lumpkin e Dess (1996) & 6 & $9 \%$ \\
\hline Yin (1994) & 6 & $9 \%$ \\
\hline Porter (1985) & 6 & $9 \%$ \\
\hline Bettis e Hitt (1995) & 6 & $9 \%$ \\
\hline Leonard-Barton (1992) & 6 & 9 \\
\hline Miller (1983) & 6 & 9 \\
\hline Eisenhardt (1989) & 6 & 9 \\
\hline
\end{tabular}

Observação: $\mathrm{n}$ = número de vezes em que o trabalho é citado na amostra; $\mathrm{q}=\%$ sobre o total da amostra.

Fonte: elaborada pelos autores com Bibexcel

\subsection{Pareamento bibliográfico e análise fatorial}

Para um melhor entendimento do pareamento bibliográfico, realizamos uma análise fatorial para identificar sob quais abordagens os autores usam Liderando a Revolução como referência. A análise fatorial exploratória agrupou as 66 obras em grupos compostos por trabalhos similares. A similaridade foi obtida pela quantidade de vezes em que dois ou mais artigos usaram uma mesma referência, ou seja, são pareados. O conceito subjacente a esta afirmação é que, quanto maior é o número de referências comuns entre trabalhos, maior é a similaridade entre eles (Vogel, \& Güttel, 2013).

Como resultado da análise fatorial, a amostra foi dividida em quatro grupos. Após leitura dos trabalhos, os grupos foram nomeados pela característica comum entre seus componentes (Serra, Ferreira, \& Almeida,
2013). Portanto, podemos afirmar que as abordagens que nortearam os trabalhos são: gestão de mudanças; empreendedorismo; estratégia e competitividade e inovação (Tabela 2).

A influência do pensamento de Gary Hamel, especificamente de Liderando a Revolução, pode ser notada, em trabalhos de grupo 1. Os artigos deste grupo usam as mudanças internas à empresa, como a implantação de um sistema ERP (McAdam, \& Galloway, 2005), tecnológicas ou ambientais (Massey, $\& \mathrm{Wu}, 2005$ ), ou ainda ocorrida no mercado (Hart \& Christensen, 2002) para sinalizar que as empresas necessitam reagir de forma diferente da usual para sobreviverem e ter bom desempenho (Cascio, 2002).

No grupo 2, com artigos sobre empreendedorismo, Wiklund e Shapherd (2003; 2005) ponderam que a orientação empreendedora das empresas tem relação positiva com o desempenho e 
Kuratko e Goldsby (2004) pregam que o empreendedorismo corporativo é uma tendência ser considerada, sendo necessária a participação de todos os funcionários. $\mathrm{O}$ empreendedorismo é uma competência essencial que algumas empresas possuem ou precisam desenvolver, no dizer de Gary Hamel, para sobreviver na era da revolução.

O grupo 3, reúne trabalhos sobre estratégia e competitividade. O artigo de Fiol (2001) afirma que, se uma vantagem competitiva estiver ancorada nas competências essenciais da empresa, ela se comportará como uma vantagem sustentável, mesmo que seja imitável por competidores. Inovações radicais são tratadas por O'Connor e Rice (2001) como forma de aumentar participação de mercado por meio do reconhecimento de oportunidades.

$\mathrm{O}$ último grupo, traz trabalhos relacionados às inovações. Por exemplo, para D’Aveni, Dagnino e Smith (2010), vivemos na era das vantagens temporárias em que as inovações precisam ocorrer uma após outra para manter a competitividade, ou O'Regan e Ghobadian (2006) para que as pequenas empresas têm a vantagem de obter mais rapidamente ganhos de suas inovações devido a sua flexibilidade e baixa inércia estrutural.

Ao analisar os resultados da Tabela 2 percebemos que a influência de Hamel (2000) tem muito peso em periódicos mais gerenciais. Há diversos artigos de periódicos como Harvard Businnes Review, Academy of Management Executive, até mesmo o Long Range Planning, que fica em um local intermediário entre um periódico de cunho mais gerencial e mais acadêmico. Tal presença nestes periódicos não é um demérito para a obra de Hamel, na verdade, mostra que o autor conseguiu fugir às barreiras acadêmicas e falar para outros públicos. Além disso, a obra apresenta um tom mais prescritivo e apresenta ideias de uma forma menos fundamentada empiricamente, quase um manifesto. Este tom apresenta-se de modo oposto a outras obras que tratam da inovação como fonte de vantagem competitiva, caso de Christensen (1997).

Tabela 2 - Resultado da análise fatorial exploratória

\begin{tabular}{|c|c|c|c|c|c|c|c|}
\hline \multirow{2}{*}{\multicolumn{2}{|c|}{$\begin{array}{c}\text { Fator } 1 \\
\text { Gestão das mudanças }\end{array}$}} & \multicolumn{2}{|l|}{ Fator 2} & \multicolumn{2}{|l|}{ Fator 3} & \multicolumn{2}{|l|}{ Fator 4} \\
\hline & & \multicolumn{2}{|c|}{ Empreendedorismo } & \multicolumn{2}{|c|}{$\begin{array}{c}\text { Estratégia e } \\
\text { competitividade }\end{array}$} & \multicolumn{2}{|l|}{ Inovação } \\
\hline $\begin{array}{c}\text { McAdam e } \\
\text { Galloway (2005) }\end{array}$ &, 811 & $\begin{array}{l}\text { Wiklund e } \\
\text { Shepherd } \\
(2003)\end{array}$ &, 858 & Fiol (2001) & ,768 & $\begin{array}{l}\text { D'Aveni, Dagnino e } \\
\text { Smith (2010) }\end{array}$ & ,772 \\
\hline $\begin{array}{l}\text { Massey e Wu } \\
\quad(2005)\end{array}$ &, 725 & $\begin{array}{l}\text { Wiklund e } \\
\text { Shepherd } \\
(2005)\end{array}$ &, 822 & Mason (2007) & ,740 & $\begin{array}{c}\text { O'Regan e Ghobadian } \\
\text { (2006) }\end{array}$ & ,665 \\
\hline $\begin{array}{l}\text { Hart e Christensen } \\
\text { (2002) }\end{array}$ & ,680 & $\begin{array}{l}\text { Kuratko e } \\
\text { Goldsby } \\
(2004)\end{array}$ &, 811 & $\begin{array}{l}\text { Mason e Leek } \\
\quad(2008)\end{array}$ & ,713 & $\begin{array}{l}\text { Ireland, Hitt, Camp e } \\
\text { Sexton (2001) }\end{array}$ & ,659 \\
\hline Cascio (2005) &, 651 & $\begin{array}{c}\text { Madsen } \\
(2007)\end{array}$ &, 805 & $\begin{array}{c}\text { O'Connor e Rice } \\
(2001)\end{array}$ & ,692 & $\begin{array}{c}\text { Dushnitsky e Lenox } \\
(2005)\end{array}$ & ,651 \\
\hline $\begin{array}{l}\text { Jung e Wang } \\
\text { (2006) }\end{array}$ &, 650 & $\begin{array}{l}\text { Kellermanns e } \\
\text { Eddleston } \\
(2006)\end{array}$ &, 791 & $\begin{array}{l}\text { Huang, Chung e } \\
\text { Lin (2009) }\end{array}$ &, 542 & $\begin{array}{c}\text { Keupp, Palmie e } \\
\text { Gassmann (2012) }\end{array}$ &, 572 \\
\hline Adler (2006) & ,646 & Wang (2008) &, 779 & $\begin{array}{c}\text { Hull e } \\
\text { Rothenberg } \\
(2008)\end{array}$ &, 528 & $\begin{array}{l}\text { Hitt, Ireland, Camp e } \\
\text { Sexton (2001) }\end{array}$ &, 536 \\
\hline $\begin{array}{l}\text { Tourish e Vatcha } \\
\qquad(2005)\end{array}$ & ,628 & $\begin{array}{c}\text { Wiklund, } \\
\text { Patzelt e } \\
\text { Shepherd } \\
(2009)\end{array}$ &, 740 & $\begin{array}{c}\text { Van Dijk e van } \\
\text { den Ende (2002) }\end{array}$ &, 525 & $\begin{array}{l}\text { McNamara, Vaaler e } \\
\text { Devers (2003) }\end{array}$ &, 529 \\
\hline $\begin{array}{l}\text { Sawhney e Zabin } \\
(2002)\end{array}$ &, 559 & $\begin{array}{l}\text { Hindle e } \\
\text { Yencken } \\
(2004) \\
\end{array}$ & ,638 & $\begin{array}{l}\text { Kiessling, et al. } \\
\text { (2009) }\end{array}$ &, 519 & $\begin{array}{c}\text { Goodale, Kuratko e } \\
\text { Hornsby (2011) }\end{array}$ & ,499 \\
\hline $\begin{array}{l}\text { Kotabe, Mol e } \\
\text { Murray (2008) }\end{array}$ &, 535 & $\begin{array}{l}\text { Judge e } \\
\text { Elenkov } \\
(2005)\end{array}$ &, 485 & $\begin{array}{l}\text { Benbya e } \\
\text { McKelvey } \\
(2006)\end{array}$ &, 517 & $\begin{array}{l}\text { Hodgkinson e Starkey } \\
\text { (2011) }\end{array}$ & ,469 \\
\hline $\begin{array}{c}\text { Stewart e } \\
\text { Nandkeolyar } \\
(2006)\end{array}$ &, 506 & $\begin{array}{l}\text { Herrmann } \\
(2005)\end{array}$ &, 458 & $\begin{array}{l}\text { Vaaler e } \\
\text { McNamara } \\
(2004)\end{array}$ &, 516 & & \\
\hline $\begin{array}{c}\text { Perretti e Negro } \\
(2006)\end{array}$ & ,490 & $\begin{array}{l}\text { Dobni e } \\
\text { Luffman }\end{array}$ &, 440 & $\begin{array}{c}\text { Bessant, et al. } \\
(2005)\end{array}$ &, 511 & & \\
\hline
\end{tabular}


Você Diz Que Quer Uma Revolução: Todos Queremos Mudar o Mundo

\begin{tabular}{|c|c|c|c|c|c|l|l|}
\hline & & $(2003)$ & & & & & \\
\hline $\begin{array}{c}\text { Elenkov, Judge e } \\
\text { Wright (2005) }\end{array}$ &, 484 & $\begin{array}{c}\text { Hannah e } \\
\text { Lester (2009) }\end{array}$ &, 417 & $\begin{array}{c}\text { Hobday, Rush e } \\
\text { Bessant (2004) }\end{array}$ &, 503 & & \\
\hline $\begin{array}{c}\text { Francis e Bessant } \\
\text { (2005) }\end{array}$ &, 469 & & & & & & \\
\hline Markides (2006) &, 455 & & & & & & \\
\hline $\begin{array}{c}\text { Hart e Sharma } \\
\text { (2004) }\end{array}$ &, 451 & & & & & & \\
\hline $\begin{array}{c}\text { Leifer e O'Connor } \\
(2001)\end{array}$ &, 435 & & & & & & \\
\hline $\begin{array}{c}\text { Whittington, et al. } \\
\text { (2003) }\end{array}$ &, 399 & & & & & \\
\hline $\begin{array}{c}\text { Variância explicada } \\
\text { 27,764\% }\end{array}$ & $9,948 \%$ & $8,746 \%$ & & $6,736 \%$ & \\
\hline
\end{tabular}

Fonte: elaborado pelos autores com SPSS, versão 20.

\subsection{Discussão}

\section{Liderando a Revolução e RBV}

As contribuições de Hamel (2000) se fazem presentes em uma variedade de abordagens. Esta característica indica que sua teoria central tem contribuições para diversos intervenientes. Em uma segunda observação, é possível identificar a forte relação entre as citações de Hamel (2000) e as teorias utilizadas em estratégia, mais especificamente a visão baseada em recursos. Esta relação sugere que Hamel (2000) pode ser um ponto para o estudo da inovação quando tangenciando a pesquisa em estratégia, para explicar como as inovações radicais desenvolvem vantagens competitivas.

Outro ponto, é que o trabalho de Hamel (2000), atua como ponte entre o paradigma SCP (structure-conduct-performance - Porter, 1980) e a RBV, uma vez que a solução proposta em sua obra é justamente para fugir dos mercados com grande concorrência, nos quais as fontes externas não ofereciam mais respostas, partindo-se então para encontrar soluções na própria organização. Inclusive, a própria emergência e relevância da obra condiz com as mudanças apresentadas em Ramos-Rodríguez \& RuízNavarro (2004).

Para Hamel (2000), a base para as inovações radicais são as competências centrais da organização. Tal afirmação é condizente com a evolução dos trabalhos deste autor e, relacionando a estratégia, mantém uma posição alinhada com a RBV. Além disso, esta afirmação faz com que as inovações radicais implementadas pela organização estejam relacionadas à dependência de caminho. Embora o conceito não esteja claramente presente no livro, as inovações de uma organização serão fruto das competências construídas por esta ao longo do tempo.

Sob esta perspectiva da dependência do caminho, a obra de Hamel (2000) apresenta ideias semelhantes ao conceito de competências de dinâmicas (Teece, Pisano, \& Shuen, 1997). Tanto que a obra de
Teece, Pisano e Shuen é a mais citada em conjunto com Hamel (2000) (Tabela 1). Porém, enquanto que a inovação radical é a chave para mercados muito competitivos, as capacidades dinâmicas, originalmente, são a solução para ambientes em constante mudança. Além disso, enquanto que, de forma velada, a dependência de caminho em Hamel (2000) define a estrutura que gera inovações radicais, em Teece, Pisano e Chuen (1997) a dependência do caminho é um dos fatores que contribuem para ganhos superiores da organização nestes ambientes.

A dependência em relação as competências construídas anteriormente também relaciona o trabalho de Hamel (2000) ao trabalho de Cohen e Levinthal (1990). Embora o foco de Hamel (2000) seja mais estreito, as inovações radicais, sua ideia depende também do conceito de capacidade absortiva no sentido de que os estímulos externos, e sua leitura e interpretação, são determinantes para o desenvolvimento de inovações radicais e que estas inovações dependem das competências construídas pela organização. Além disso, a capacidade dinâmica, bem como o foco em inovações radicais, deve ser entendida a nível organizacional.

\section{Liderando a Revolução e Inovação}

É importante ressaltar também a conversa de Hamel (2000) com outros temas, dentre eles, a inovação. O papel transformador da inovação radical já foi salientado em Schumpeter (1934). Inclusive, em Schumpeter a inovação radical é a fonte de transformações no mercado, tirando-o da posição de equilíbrio. No entanto, em Hamel (2000) a inovação radical, ganha um papel de fonte de vantagem competitiva, não como elemento transformador do mercado. Outra diferença importante em relação a obra de Schumpeter, é que em Hamel (2000), o ator que busca e implementa inovação radical não é e nem pode ser o empreendedor individual, mas sim a organização como um todo, pois esta deve se estruturar com 
competências, pessoas e processos, para continuamente lançar inovações radicais.

Outra obra que pode exercer um interessante diálogo com Hamel (2000), é a obra de Christensen (1997), nela a inovação também é fonte de vantagem competitiva, porém, neste caso, trata-se da inovação disruptiva, que vem muitas vezes sendo usada como sinônimo de inovação radical. Inclusive, cabe aqui definir a inovação disruptiva que, segundo Christensen (1997), pode ter um desempenho abaixo do mercado, mas deve gerar valor para o cliente por meio de produtos mais baratos, simples e mais convenientes para o uso. A inovação radical de Hamel (2000), por sua vez, também gera valor para o cliente, no entanto, deve também gerar riqueza para a organização, por meio de produtos que mudem as "expectativas dos clientes, alterem a economia da indústria, e redefinam as bases para a vantagem competitiva" (p. 62). Por esta definição, uma inovação disruptiva pode ser radical, mas não necessariamente será.

A obra de Christensen (1997) também foca-se na mudança de modelo negócio, porém enquanto para a Christensen o objetivo do modelo de negócio é oferecer produtos existentes mais de formas mais simples e mais baratas. Para Hamel, o modelo de negócio é o resultado das inovações radicais, de modo que o modelo de negócio possibilite a organizações inovar em todos os seus níveis e transacionar sua inovação.

Aqui cabe uma reflexão, pelo próprio tom do livro Hamel (2000), apresenta-se como um manifesto para a inovação radical, esta é apresentada como a chave para a vantagem competitiva. Contudo, vale ressaltar que a inovação radical, normalmente, é um processo muito custoso e que envolve risco. Inclusive, este risco é reconhecido por Hamel ao longo do livro. Por isso é sugerido que a organização seja construída para sustentar diversas inovações radicais, não apenas uma. Porém, ainda é necessário atentar para estas questões. Christensen (1997), por exemplo, pontua exemplos de inovações disruptivas que, apesar do modificar o mercado, inicialmente tiveram desempenho inferior aos produtos dominantes. Por outro lado, Hamel, talvez pelo tom de manifesto, diminua um pouco estes riscos e apresente soluções um tanto incertas para mitigar estes riscos.

\section{Liderando a Revolução e Teorias Emergentes em Estratégia}

De acordo com Ramos-Rodríguez e RuízNavarro (2004) e Serra, Ferreira, Almeida e Vanz (2012) há outras teorias que ganharam espaço em estratégia recentemente. Dentre elas, podemos destacar a Teoria dos Custos de Transação e Teoria Institucional. Por ser baseado dentro do paradigma da RBV, é esperado que a obra de Hamel tenha alguns conflitos com estas teorias. No entanto, do confronto entre o trabalho de Hamel e estas teorias pode oferecer algumas oportunidades de discussão.
Inicialmente, é possível analisar as ideias e Hamel sob a luz da ecologia populacional proposta por Nelson e Winter (1982). Tanto que estas obras são citadas em conjunto em diversos trabalhos (ver Tabela 1). Pela visão de Hamel o ambiente apenas pressiona a organização para inovações, mas cabe a empresa incorporar a filosofia que deve inovar radicalmente constantemente para manter a vantagem a competitiva, assim o meio é coadjuvante neste processo, não o ator que seleciona as inovações, perpetuando modelos que sobreviverão, como proposto em Nelson \& Winter (1982)

Outro contraponto teórico atual com Hamel (2000) é com o neoinstitucionalismo. Enquanto que para Hamel ser igual prejudica a empresa, para a visão neo-institucional ser diferente pode prejudicar a empresa e limitar seu acesso a recursos, pois adotar estruturas organizacionais diferentes pode prejudicar a legitimidade da organização e seu acesso a recursos (Meyer \& Rowan, 1977). Esta última poderá nos dizer sobre a necessidade da se legitimar socialmente a inovação para que esta gere vantagem competitiva (Bitektine \& Haack, 2015; Zucker, 1989). Por último, ainda sob a teoria institucional, pode-se analisar como estas inovações são absorvidas e replicadas pelo mercado sob o ponto de vista do isomorfismo (DiMaggio \& Powell, 1983) ou, por outro lado, como as empresas fugirão da necessidade de se adequarem as pressões institucionais geradas por estas inovações (Meyer \& Rowan, 1977).

O próprio Hamel cita os custos de transação de implementar uma inovação radical em sua obra, o autor reconhece que há elevados custos de transação em criar e implementar inovações radicais. No entanto, e indo ao encontro de Hamel (2000), uma forma de melhorar os custos de transação é a criação de competências e capabilities que diminuam estes custos ao longo da cadeia e ajudem na tomada de decisão de make or buy (Jacobides \& Winter, 2005). No entanto, os custos relacionados a questões de governança e ou da falta de legitimidade do novo ainda permanece. Assim, a Teoria de Custos de Transação pode ajudar no diálogo da obra de Hamel com questões envolvendo tanto RBV quanto Teoria institucional.

A linha teórica de Hamel (2000) tem o potencial de trazer estudos futuros para diversas disciplinas. Por unir duas importantes áreas (inovação e estratégia) sob a óptica das inovações radicais, Hamel (2000) pode ser melhor explorado em três vertentes. Primeiro, o livro pode ser empregado nas análises históricas de sobrevivência e declínio. Empresas como Kodak e Olivetti tiveram a oportunidade de empregar inovações radicais em seus negócios, porém, optaram por continuar em suas perspectivas incrementais e falharam. A inter-relação entre inovação e estratégia fica bem evidente no estudo de casos históricos utilizando Hamel (2000). Em segundo lugar, é possível explicar em parte o movimento macroeconômico que segue uma inovação radical, como por exemplo as influências da Internet na competitividade das 
empresas. Um terceiro ponto para a pesquisa futura seria a utilização de Hamel (2000) para auxiliar nas discussões ligadas ao desenvolvimento de inovação radical dentro das empresas, de forma a trazer novas explicações para o posicionamento dos departamentos de pesquisa e desenvolvimento de acordo com as estratégias de inovação empregadas.

\section{CONCLUSÃO}

Este trabalho teve por objetivo pesquisar o pensamento de Gary Hamel por meio de algumas de suas obras e, especificamente, verificar a influência de Liderando a Revolução nas obras de outros autores. Para tal, foi obtida uma amostra de trabalhos que citaram Liderando a Revolução e, por meio de análises bibliométricas, pudemos constatar que o uso deste livro, publicado em 2000, permaneceu constante dentro do período amostrado, de 2001 a 2015. Identificamos que sua obra é referenciada em artigos sobre gestão de mudanças, empreendedorismo, estratégia e inovações.

\section{REFERÊNCIAS}

Adler, N. (2006). The Arts \& Leadership: Now that we can do anything, what will we do? Academy of Management Learning \& Education, 5(4), 486-499.

Barney, J. (1991). Firm Resources and Sustained Competitive Advantage. Journal of Management, 17(1), 99-120.

Benbya, H., \& McKelvey, B. (2006). Using coevolutionary and complexity theories to improve IS alignment: a multi-level approach. Journal of Information technology, 21(4), 284-298.

Bessant, J., Lamming, R., Noke, H., \& Phillips, W. (2005). Managing innovation beyond the steady state. Technovation, 25(12), 1366-1376.

Bettis, R., \& Hitt, M. (1995). The new competitive landscape. Strategic Management Journal, 16(S1), 7-19.

Bitektine, A., \& Haack, P. (2015). The "macro" and the "micro" of legitimacy: Toward a multilevel theory of the legitimacy process. Academy of Management Review, 40(1), 49-75.

Cascio, W. (2005). Strategies for responsible restructuring. The Academy of Management Executive, 19(4), 39-50.

Christensen, C. M. (1997). Innovator's Dilemma: When New Technologies Cause Great Firms to Fail. Harvard Business Review Press.
Cohen, W. M., \& Levinthal, D. A. (1990). Absorptive Capacity: A New Perspective on Learning and Innovation. Administrative Science Quarterly, 35(1), 128 .

Covin, J., \& Slevin, D. (1989). Strategic management of small firms in hostile and benign environments. Strategic Management Journal, 10(March 1987), 75-87.

D'Aveni, R., Dagnino, G., \& Smith, K. (2010). The age of temporary advantage. Strategic Management Journal, 31(13), 1371-1385.

DiMaggio, P. J., \& Powell, W. W. (1983). The iron cage revisited: Institutional isomorphism and collective rationality in organizational fields. American Sociological Review, 48(2), 147-160.

Dobni, C., \& Luffman, G. (2003). Determining the scope and impact of market orientation profiles on strategy implementation and performance. Strategic management journal, 24(6), 577-585.

Dushnitsky, G., \& Lenox, M. (2005). When do firms undertake R\&D by investing in new ventures? Strategic Management Journal, 26(10), 947-965.

Eisenhardt, K. (1989). Building Theories from Case Study Research. Academy of Management Review, 14(4), 532-550.

Eisenhardt, K., \& Martin, J. (2000). Dynamic capabilities: what are they? Strategic Management Journal, 21(10-11), 1105-1121.

Elenkov, D., Judge, W., \& Wright, P. (2005). Strategic leadership and executive innovation influence: an international multi-cluster comparative study. Strategic Management Journal, 26(7), 665-682.

Fiol, C. (2001). Revisiting an identity-based view of sustainable competitive advantage. Journal of Management, 27(6), 691-699.

Francis, D., \& Bessant, J. (2005). Targeting innovation and implications for capability development. Technovation, 25(3), 171-183.

Goodale, J., Kuratko, D., Hornsby, J., \& Covin, J. (2011). Operations management and corporate entrepreneurship: The moderating effect of operations control on the antecedents of corporate entrepreneurial activity in relation to innovation performance. Journal of Operations Management, 29(1), 116-127.

Hamel, G. (2000). Leading the revolution: How to thrive in turbulent times by making innovation a way of life. Boston, MA: Harvard Business School Press. 
Hamel, G., \& Prahalad, C. K. (1994). Competing for the Future. Harvard Business Press.

Hannah, S. T., \& Lester, P. B. (2009). A multilevel approach to building and leading learning organizations. The Leadership Quarterly, 20(1), 3448.

Hart, S., \& Christensen, C. (2002). The great leap: Driving innovation from the base of the pyramid. MIT Sloan management review, 44(1), 51.

Hart, S., \& Sharma, S. (2004). Engaging fringe stakeholders for competitive imagination. The Academy of Management Executive, 18(1), 7-18.

Herrmann, P. (2005). Evolution of strategic management: the need for new dominant designs. International Journal of Management Reviews, 7(2), 111-130.

Hindle, K., \& Yencken, J. (2004). Public research commercialisation, entrepreneurship and new technology based firms: an integrated model. Technovation, 24(10), 793-803.

Hitt, M., Ireland, R., Camp, S., \& Sexton, D. (2001). Strategic entrepreneurship: Entrepreneurial strategies for wealth creation. Strategic management journal, 22(6-7), 479-491.

Hobday, M., Rush, H., \& Bessant, J. (2004). Approaching the innovation frontier in Korea: the transition phase to leadership. Research policy, 33(10), 1433-1457.

Hodgkinson, G. P., \& Starkey, K. (2011). Not simply returning to the same answer over and over again: reframing relevance. British Journal of Management, 22(3), 355-369.

Huang, Y., Chung, H., \& Lin, C. (2009). R\&D sourcing strategies: determinants and consequences. Technovation, 29(3), 155-169.

Hull, C. E., \& Rothenberg, S. (2008). Firm performance: The interactions of corporate social performance with innovation and industry differentiation. Strategic Management Journal, 29(7), 781-789.

Ireland, R., Hitt, M., Camp, S., \& Sexton, D. (2001). Integrating entrepreneurship and strategic management actions to create firm wealth. The Academy of Management Executive, 15(1), 49-63.

Jacobides, M. G., \& Winter, S. G. (2005). The coevolution of capabilities and transaction costs: Explaining the institutional structure of production.
Strategic Management Journal, 26(5), 395-413.

Judge, W., \& Elenkov, D. (2005). Organizational capacity for change and environmental performance: an empirical assessment of Bulgarian firms. Journal of Business Research, 58(7), 893901.

Jung, J., \& Wang, Y. (2006). Relationship between total quality management (TQM) and continuous improvement of international project management (CIIPM). Technovation, 26(5), 716-722.

Kellermanns, F., \& Eddleston, K. (2006). Corporate entrepreneurship in family firms: A family perspective. Entrepreneurship theory and practice, 30(6), 809-830.

Keupp, M., Palmié, M., \& Gassmann, O. (2012). The strategic management of innovation: A systematic review and paths for future research. International Journal of Management Reviews, 14(4), 367-390.

Kiessling, T., Richey, R., Meng, J., \& Dabic, M. (2009). Exploring knowledge management to organizational performance outcomes in a transitional economy. Journal of World Business, 44(4), 421-433.

Kotabe, M., Mol, M., \& Murray, J. (2008). Outsourcing, performance, and the role of ecommerce: A dynamic perspective. Industrial Marketing Management, 37(1), 37-45.

Kuratko, F., \& Goldsby, M. (2004). Corporate entrepreneurs or rogue middle managers? A framework for ethical corporate entrepreneurship. Journal of Business Ethics, 55(1), 13-30.

Leifer, R., O'Connor, G. C., \& Rice, M. (2001). Implementing radical innovation in mature firms: The role of hubs. The Academy of Management Executive, 15(3), 102-113.

Leonard-Barton, D. (1992). Core capabilities and core rigidities: A paradox in managing new product development. Strategic Management Journal, 13(S1), 111-125.

Lumpkin, G., \& Dess, G. (1996). Clarifying the Entrepreneurial Orientation Construct and Linking It to Performance. The Academy of Management Review, 21(1), 135-172.

Madsen, E. (2007). The significance of sustained entrepreneurial orientation on performance of firms-A longitudinal analysis. Entrepreneurship and Regional Development, 19(2), 185-204.

March, J. (1991). Exploration and exploitation in organizational learning. Organization Science, 2(1), 
71-87.

Markides, C. (2006). Disruptive innovation: In need of better theory. Journal of product innovation management, 23(1), 19-25.

Mason, K., \& Leek, S. (2008). Learning to build a supply network: an exploration of dynamic business models. Journal of Management Studies, 45(4), 774-799.

Mason, R. (2007). The external environment's effect on management and strategy: a complexity theory approach. Management Decision, 45(1), 10-28.

Massey, C., \& Wu, G. (2005). Detecting regime shifts: The causes of under-and overreaction. Management Science, 51(6), 932-947.

McAdam, R., \& Galloway, A. (2005). Enterprise resource planning and organisational innovation: a management perspective. Industrial Management \& Data Systems, 105(3), 280-290.

McNamara, G., Vaaler, P., \& Devers, C. (2003). Same as it ever was: The search for evidence of increasing hypercompetition. Strategic Management Journal, 24(3), 261-278.

Meyer, J. W., \& Rowan, B. (1977). Institutionalized Organizations: Formal Structure as Myth and Ceremony. American Journal of Sociology, 83(2), 340-363.

Miller, D. (1983). The correlates of entrepreneurship in three types of firms. Management Science, 29(7), $770-793$.

Miller, D., \& Friesen, P. (1982). Innovation in conservative and entrepreneurial firms: Two models of strategic momentum. Strategic Management Journal, 3(1), 1-25.

Nelson, R., \& Winter, S. (1982). An Evolutionary Theory of Economic Change. Cambridge, MA: The Belknap Press.

O'Connor, G., \& Rice, M. (2001). Opportunity recognition and breakthrough innovation in large established firms. California Management Review, 43(2), 95-116.

O'Regan, N., Ghobadian, A., \& Sims, M. (2006). Fast tracking innovation in manufacturing SMEs. Technovation, 26(2), 251-261.

Penrose, E. (1959). The Theory of the Growth of the Firm. White Plains, NY: M.E. Sharpe.

Perretti, F., \& Negro, G. (2006). Filling empty seats: How status and organizational hierarchies affect exploration versus exploitation in team design. Academy of Management Journal, 49(4), 759-777.

Pilkington, A. (2006). Bibexcel-Quick Start Guide to Bibliometrics and Citation Analysis.

Porter, M. (1980). Competitive Strategy. New York: Free Press - Simon \& Schuster Inc.

Porter, M. (1985). Competitive Advantage. New York: Free Press.

Prahalad, C., \& Hamel, G. (1990). The core competence of the corporation. Harvard Business Review, may-june, 79-91.

Ramos-Rodríguez, A.-R., \& Ruíz-Navarro, J. (2004). Changes in the intellectual structure of strategic management research: a bibliometric study of the Strategic Management Journal, 1980-2000. Strategic Management Journal, 25(10), 981-1004.

Sawhney, M., \& Zabin, J. (2002). Managing and measuring relational equity in the network economy. Journal of the Academy of Marketing Science, 30(4), 313-332.

Schumpeter, J. A. (1934). The theory of economic development: An inquiry into profits, capital, credit, interest, and the business cycle. Transaction Publishers.

Serra, F. A. R., Ferreira, M. A. S. P. V., Almeida, M. I. R. de, \& Vanz, S. A. de S. (2012). A pesquisa em Administração estratégica nos primeiros anos do século XXI: um estudo bibliométrico de citação e cocitação no Strategic Management Journal entre 2001 e 2007. Revista Eletrônica de Estratégia \& Negócios, 5(2), 257-274.

Shane, S., \& Venkataraman, S. (2000). The promise of entrepreneurship as a field of research. Academy of Management Review, 25(1), 217-227.

Stewart, G., \& Nandkeolyar, A. (2006). Adaptation and intraindividual variation in sales outcomes: Exploring the interactive effects of personality and environmental opportunity. Personnel Psychology, 59(2), 307-332.

Teece, D. J. (2010). Business Models, Business Strategy and Innovation. Long Range Planning, 43(2-3), 172-194.

Teece, D. J., Pisano, G., \& Shuen, A. (1997). Dynamic capabilities and strategic management. Strategic Management Journal, 18(7), 509-533.

Tourish, D., \& Vatcha, N. (2005). Charismatic leadership and corporate cultism at Enron: The 
elimination of dissent, the promotion of conformity and organizational collapse. Leadership, 1(4), 455480.

Utterback, J. (1994). Mastering the dynamics of innovation: how companies can seize opportunities in the face of technological change. University of Illinois at Urbana-Champaign's Academy for Entrepreneurial Leadership Historical Research Reference in Entrepreneurship.

Vaaler, P. M., \& McNamara, G. (2004). Crisis and competition in expert organizational decision making: Credit-rating agencies and their response to turbulence in emerging economies. Organization Science, 15(6), 687-703.

Van Dijk, C., \& Van Den Ende, J. (2002). Suggestion systems: transferring employee creativity into practicable ideas. $R \& D$ Management, 32(5), 387395.

Vogel, R., \& Güttel, W. (2012). The dynamic capability view in strategic management: A bibliometric review. International Journal of Management Reviews, 15(4), 426-446.

Wang, C. (2008). Entrepreneurial orientation, learning orientation, and firm performance. Entrepreneurship Theory and Practice, 32(4), 635657.

Wernerfelt, B. (1984). A resource-based view of the firm. Strategic Management Joumal, 5(2), 171180.

Whittington, R., Jarzabkowski, P., Mayer, M., Mounoud, E., Nahapiet, J., \& Rouleau, L. (2003).
Taking strategy seriously responsibility and reform for an important social practice. Journal of Management Inquiry, 12(4), 396-409.

Wiklund, J., \& Shepherd, D. (2003). Knowledge-based resources, entrepreneurial orientation, and the performance of small and medium-sized businesses. Strategic management journal, 24(13), 1307-1314.

Wiklund, J., \& Shepherd, D. (2005). Entrepreneurial orientation and small business performance: a configurational approach. Journal of business venturing, 20(1), 71-91.

Wiklund, J., Patzelt, H., \& Shepherd, D. (2009). Building an integrative model of small business growth. Small Business Economics, 32(4), 351-374.

Yin, R. (1994). Case study research: Design and methods. Beverly Hills: Sage Publishing.

Zahra, S. (1991). Predictors and financial outcomes of corporate entrepreneurship: An exploratory study. Journal of business venturing, 6(4), 259-285.

Zahra, S., \& Covin, J. (1995). Contextual influences on the corporate entrepreneurship-performance relationship. Journal of Business Venturing, 10(1), 43-59.

Zucker, L. G. (1989). Combining Institutional Theory and Population Ecology: No Legitimacy, No History. American Sociological Review, 54(4), 542.

Zupic, I, Cater, T. (2015). Bibliometric Methods in Management and Organization. Organization Research Methods, 18(3), 429-472. 